\title{
EL FENÓMENO DE CAMBIO CLIMÁTICO EN LA PERCEPCIÓN DE LA COMUNIDAD INDÍGENA PURÉPECHA DEL MUNICIPIO DE CHILCHOTA, MICHOACÁN, MÉXICO
}

\author{
Sandra Llovizna GONZÁLEZ MARTÍNEZ ${ }^{1}$, José Teodoro SILVA GARCÍA ${ }^{1 *}$, \\ Luis Arturo ÁVILA MELÉNDEZ ${ }^{1}$, Rodrigo MONCAYO-ESTRADA ${ }^{2}$, \\ Gustavo CRUZ CÁRDENAS ${ }^{1}$ y Luís Fernando CEJA TORRES ${ }^{1}$
}

${ }^{1}$ Centro Interdisciplinario de Investigación para el Desarrollo Integral Regional, Unidad Michoacán, Instituto Politécnico Nacional. Justo Sierra 28, Jiquilpan, Michoacán, C. P. 59510

${ }^{2}$ Centro Interdisciplinario de Ciencias Marinas, Instituto Politécnico Nacional. Avenida Instituto Politécnico Nacional, Colonia Playa Palo de Santa Rita, La Paz, Baja California Sur, México, C. P. 23096

*Autor para correspondencia: tsilva09@hotmail.com

(Recibido junio 2016; aceptado marzo 2017)

Palabras clave: adaptación climática, elementos causales, análisis multivariado

\section{RESUMEN}

En esta investigación se planteó como objetivo evaluar la percepción o conocimiento que tiene respecto al fenómeno de cambio climático la población indígena Purépecha del Municipio de Chilchota, Michoacán, México. Lo anterior, sin dejar de reconocer algunos mecanismos de adaptación basados en su cultura, tradiciones y conocimientos actuales. Como instrumento para obtener información se aplicaron encuestas. Un total de 298 fueron realizadas y distribuidas en cuatro comunidades del municipio: Zopoco (49), Tanaquillo (50), Carapan (59), Ichan (37) y la cabecera municipal Chilchota (103). Se aplicaron técnicas multivariadas para el análisis de los datos obtenidos de las encuestas, que permitieran reducir la estructura de datos y la clasificación de las variables en grupos. Los resultados muestran que la población indígena del municipio de Chilchota percibe cambios locales en el clima atribuidos a diversas acciones que el ser humano ha venido desarrollando en los últimos años (tala de árboles, quema de basura, contaminación, etc.), no asociando dichas variaciones de manera inmediata con el fenómeno de cambio climático. Como una medida de adaptación a los cambios percibidos, los calendarios agrícolas de siembra de la región se han ajustado. Estos cambios climáticos coinciden con el comportamiento cuantitativo para el periodo 1968-2007, de la temperatura media anual y de la precipitación. En dicho periodo la primera registra un incremento de $0.5^{\circ} \mathrm{C}$, mientras que para la segunda se reportan $150 \mathrm{~mm}$ anuales menos.

Key words: climate adaptation, causal factors, multivariate analysis

\begin{abstract}
The aim of this research was to estimate the perception or knowledge about the phenomenon of climate change in the Purepecha's indigenous population in the Municipality of Chilchota, Michoacan, Mexico. The latter, taking into account some adaptation mechanisms that the stakeholders may have, based on their current culture, traditions
\end{abstract}


and knowledge. Different surveys were applied as a tool to obtain information. A total of 298 surveys were carried out and distributed in four communities of the municipality: Zopoco (49), Tanaquillo (50), Carapan (59), Ichan (37) and the municipal head Chilchota (103). Multivariate techniques were applied on the surveys to reduce the data structure into groups of variables. Results show that the indigenous population of the Chilchota municipality perceives local changes in the climate attributed to several anthropogenic actions developed in recent years (logging, trash burning, pollution, etc.). They do not associate such changes with the phenomenon of climate change. Agricultural planting schedules in the region have been adjusted as an adaptive measure to the perceived changes. These climate changes are consistent with the average annual temperature and precipitation behavior for the 1968-2007 periods. For this period the temperature showed an increase of $0.5^{\circ} \mathrm{C}$, meanwhile precipitation was reduced to $150 \mathrm{~mm}$ per year.

\section{INTRODUCCIÓN}

El cambio climático (CC) constituye actualmente un tema relevante, tanto en la agenda política como en la opinión pública y ha sido planteado por algunos autores como la cuestión señera del siglo XXI (Giddens 2010). El panel intergubernamental sobre cambio climático (IPCC, por sus siglas en inglés), advirtió en su primer informe que la migración humana podría ser una de las consecuencias más graves del CC (IPCC 1990). Este mismo grupo en su segundo informe planteó que "el balance de las evidencias sugiere que hay una influencia humana discernible en el cambio global" (IPCC 1995).

La contribución del grupo de trabajo III al quinto informe de evaluación (GTIII IE5) del IPCC (2014), evalúa las publicaciones sobre los aspectos científicos, tecnológicos, ambientales, económicos y sociales de la mitigación del CC.

Hace 30 años la mayoría de los climatólogos eran escépticos acerca de la naturaleza antrópica del CC. Hoy en día, reconocen una evidente huella humana en el intenso CC ocurrido en los últimos cincuenta años (Arnell 1999, Beniston 2003, Barnett et al. 2005, Altieri 2008). Existen evidencias del impacto del CC en los pasados 30 años sobre los ecosistemas de todo el planeta. Los efectos son visibles, por ejemplo, las temperaturas árticas invernales han llegado a aumentar hasta $5^{\circ} \mathrm{C}$ en algunas zonas, lo que supone siete veces la media del incremento medio mundial (AEMA 2015). Walther (2002), por su parte, reporta claras afectaciones a la fenología de los organismos, la distribución de las especies y la composición y dinámica de las comunidades.

México no es una excepción. Su situación geográfica, condiciones climáticas, orográficas e hidrológicas, entre otros factores, contribuyen a que el país sea una de las zonas más vulnerables del mundo por el CC. En la actualidad, se dan cita en este territorio una gran variedad de alteraciones provocadas por el $\mathrm{CC}$ que, en muchos casos, no son más que el inicio de una tendencia de impactos que se verán exacerbados a lo largo del siglo si no adoptamos las medidas necesarias (SEMARNAT-CICC 2007). Estudios previos de CC para México (Gay et al. 2000, 2006), llegaron a la conclusión de que el país sería propenso a experimentar temperaturas más altas y, por lo tanto, las tasas de evaporación se incrementarían. Se ha invertido una enorme cantidad de tiempo y esfuerzo para determinar los efectos meteorológicos del CC. Sin embargo, se han dedicado menos esfuerzos y recursos para analizar empíricamente las consecuencias de este CC sobre las poblaciones humanas y en especial, sobre las comunidades rurales e indígenas (Brown 2008).

En la literatura científica sobre CC, se están comenzando a incorporar evidencias de cómo las comunidades indígenas perciben el fenómeno (Marzano 2006, Salick 2007, Ishaya 2008, Osbahr et al. 2008, Byg et al. 2009, Deressa et al. 2011). Vedwan y Rhoades (2001) sugieren que en general los campesinos perciben con mayor facilidad los cambios en el clima y pueden entender mejor las causas y consecuencias locales de estos cambios. Por otra parte, McDowell (2010) refiere la vulnerabilidad de las comunidades rurales e indígenas en el contexto de los impactos potenciales del CC sobre el recurso agua y cuáles son los mecanismos de adaptación que están desarrollando. Nielsen y Reenberg (2010) plantean que la adaptación al CC en comunidades rurales e indígenas se encuentra ligada con factores económicos y culturales que influyen en la toma de decisiones de las personas para adaptarse o no. Por su parte Madisson (2006) menciona que la adaptación al CC implica un proceso de dos etapas: en la primera, el productor o campesino tiene que percibir que están ocurriendo alteraciones en su entorno ambiental relacionados con el CC y sólo a partir de dicha percepción es que se da lugar a la segunda etapa, en 
la cual el productor o campesino decide si adopta o no una medida al respecto.

En México, los pueblos indígenas demandaron su participación en toda acción de protección ambiental del planeta, así como su interés por revertir las condiciones actuales del CC (SEMARNAT-CCDS 2010).

En esta investigación se planteó como objetivo estimar cuál es la percepción de la comunidad indígena Purépecha en el Municipio de Chilchota respecto al fenómeno de CC.

El diseño del método se programó para aproximarse a dicha percepción desde dos ámbitos: por un lado, a través de la percepción de alteraciones evidentes en el clima local y por otro, a partir del conocimiento que tienen las comunidades indígenas sobre el concepto de CC.

\section{METODO}

El municipio de Chilchota se localiza al noroeste del estado de Michoacán, México, en las coordenadas $19^{\circ} 51^{\prime}$ de latitud norte y $101^{\circ} 87^{\prime}$ de longitud oeste
(Fig. 1), a una altura de $1770 \mathrm{msnm}$. Su superficie es de $305.13 \mathrm{~km}^{2}$ y representa $0.51 \%$ de la superficie del estado (SEDESOL-CONEVAL 2015). El clima es templado con una precipitación pluvial media anual de $990 \mathrm{~mm}$ y temperaturas que oscilan de $2.5 \mathrm{a}$ $38^{\circ} \mathrm{C}$ (García 1973).

Según la encuesta intercensal realizada por el INEGI en 2015, en el municipio de Chilchota se registra una población de 39035 hab, de los cuales $61 \%$ son indígenas purépechas.

Como instrumento de obtención de información, se diseñó y aplicó una encuesta (Cuadro I). En dicha encuesta se propuso investigar el nivel de percepción de los pobladores acerca de las alteraciones climáticas percibidas, relacionadas con factores como la temperatura, lluvias, heladas, vientos y sequía, sin generar una expectativa o indicio de interés por el término $\mathrm{CC}$.

La aplicación de las encuestas se realizó al azar de manera oral y directa, se visitó al encuestado en su casa (previa cita). El conjunto final de preguntas permitió captar información acerca de lo que el encuestado entendía por CC y algunas opiniones acerca de sus causas y efectos. Adicionalmente, se

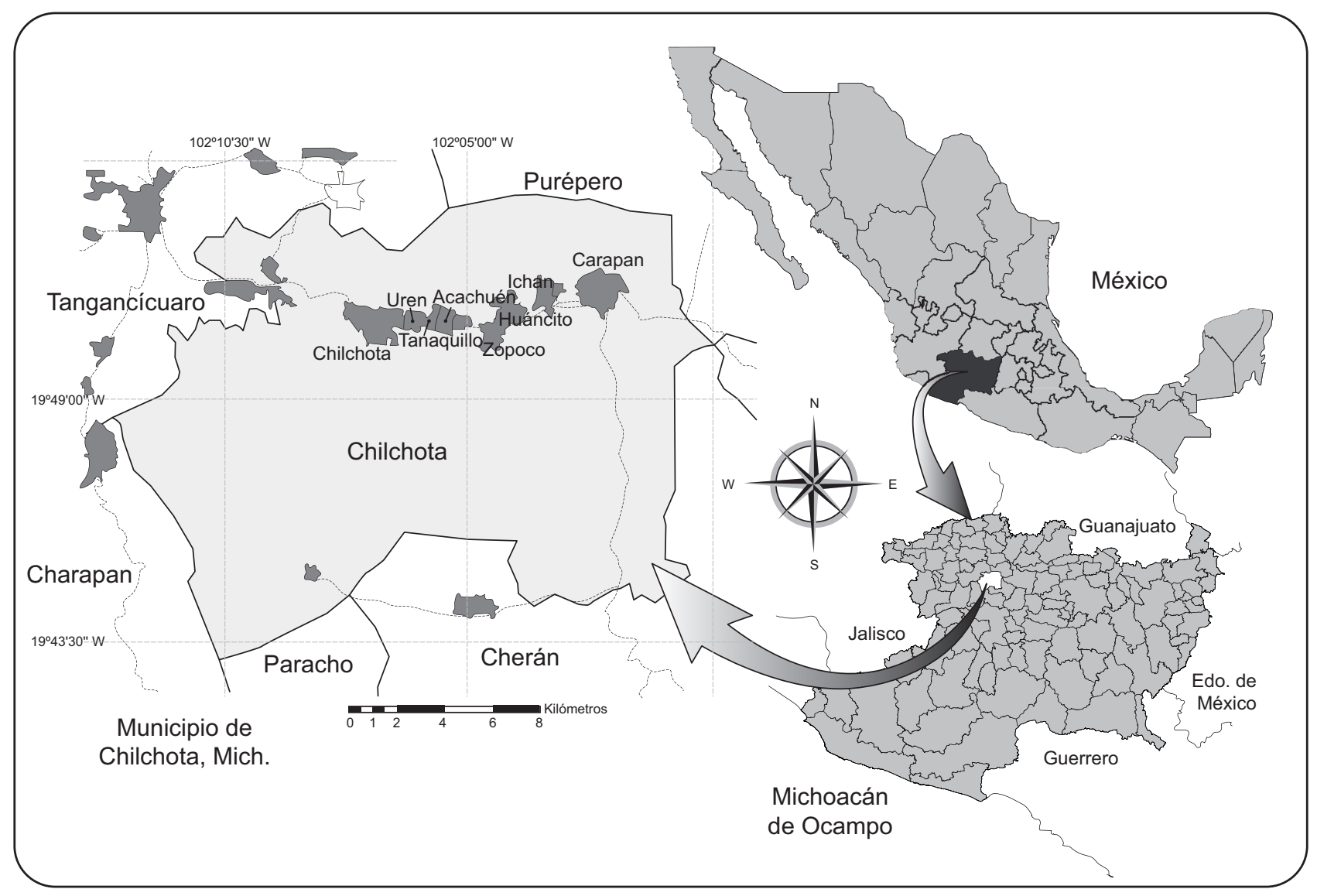

Fig. 1. Región de estudio. Municipio de Chilchota, Michoacán, México 
CUADRO I. FORMATO DE ENCUESTA APLICADA

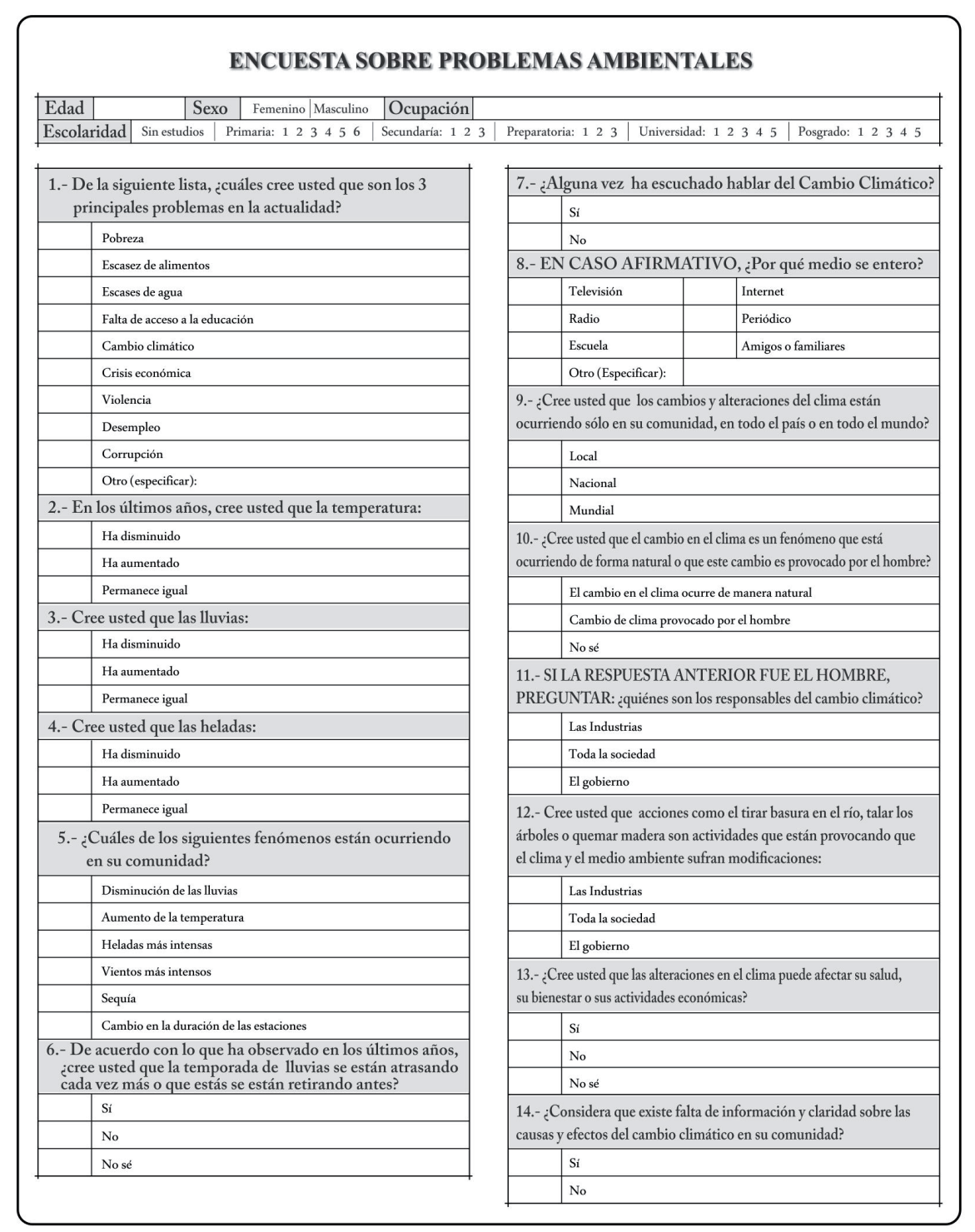

realizaron 23 entrevistas semiestructuradas a agricultores de temporal en la región. De ellas se obtuvo información respecto a la percepción de alteraciones locales del clima.

Un total de 298 encuestas fueron realizadas a igual número de personas y distribuidas en cinco comunidades del municipio de Chilchota: Zopoco (49), Tanaquillo (50), Carapan (59), Ichan (37) y la cabecera municipal Chilchota (103). Al interior del municipio indígena de Chilchota hay heterogeneidad en las proporciones de hablantes de lengua indígena (HLI) por localidad. Se seleccionaron tres comunidades con elevados porcentajes de HLI (Zopoco, Carapan e Ichan) y dos con porcentajes bajos (Chilchota y Tanaquillo). En conjunto, son representativas de la denominada "cañada de los once pueblos", corredor indígena purépecha de la región.

Se llevó a cabo un análisis estadístico de tipo estratificado y sistemático. En el estratificado, el carácter determinador fue el tamaño poblacional de las comunidades, mientras que el sistemático fue aplicable en virtud de que los elementos de la población sobre la que se efectuó el muestreo para que cubriera perfiles similares (agricultor, ama de casa, profesionista, etc.).

Se realizó un análisis de componentes principales categóricos (CATPCA, por sus siglas en inglés), cuyo 
objetivo fue la reducción del conjunto original de variables a un conjunto más reducido de componentes no correlacionados, que representen la mayor parte de la información encontrada en las variables originales (Linting et al. 2012).

Para la percepción del CC se aplicó un modelo confirmatorio, con el cual se identificaron y seleccionaron las variables más explicativas con la menor pérdida de información posible (Linting et al. 2012). Dichas variables tienen esa cualidad, debido a que incorporan la mayor cantidad de la varianza que presenta la matriz de datos, es decir, son las que la describen mejor (Manisera et al. 2010). Para cuantificar la varianza, el CATPCA convierte cada categoría en un valor numérico de acuerdo con el nivel de análisis de la variable por medio del escalamiento óptimo. La estimación del modelo y el escalamiento óptimo se alterna a través de un algoritmo iterativo, que converge en un punto estacionario donde la óptima cuantificación de las categorías no cambia (Manisera et al. 2010).

Uno de los resultados que presenta el CATPCA es una matriz con los coeficientes de correlación de Pearson de las variables transformadas. La magnitud y el signo del coeficiente determinan el grado y sentido de la relación entre las variables explicativas. Para determinar qué variables tienen mayor influencia, se analiza su relación con la dimensión de la solución (Zeijl et al. 2000), de esta manera se puede proporcionar una explicación robusta de la información.

Para nuestro caso se analizaron 20 variables (Cuadro II) entre las que se destacan: edad, género, ocupación, escolaridad, localidad, hablante de lengua indígena, etc. La mayoría de estas variables presentan una escala nominal (nombres) y algunas fueron convertidas a dicotómicas (en dos partes). Solamente una es de intervalos ya que se trata de la edad.

En el resumen del modelo un aspecto importante a considerar fue el valor alfa de Cronbach, que representa una medida de la consistencia interna, esto es, qué tan cercanamente relacionados están un cumulo de variables como grupo (un valor $\geq 0.7 \mathrm{se}$ considera "aceptable" en investigación relacionada a las ciencias sociales). La primera dimensión y la combinación de las dos dimensiones (total), son las que mejor explican las características de la información (Cronbach 1951).

Adicionalmente, se llevó a cabo un análisis estadístico del comportamiento de la precipitación y la temperatura en la región para un periodo de 30 años (1968-2007). Lo que sirvió para comparar los posibles cambios de esas variables en ese lapso de tiempo con la percepción de dichos cambios en las personas encuestadas. Los datos considerados se tomaron de la información generada en tres estaciones climatológicas ubicadas dentro de la zona de estudio (Cumuato, Orandino y Carapan), mismas que pertenecen a la Comisión Nacional del Agua.

\section{RESULTADOS}

La edad promedio de los encuestados fue de 40 años, $60 \%$ fueron mujeres y $40 \%$ hombres. Dado que la encuesta se aplicó en los hogares, al parecer el horario de trabajo de los hombres fuera de casa se reflejó en una sobrerrepresentación de las mujeres en la muestra, pues a escala municipal los porcentajes correspondientes de la población de 18 a 70 años son $54 \%$ mujeres y $46 \%$ hombres. En términos de escolaridad: $8 \%$ sin escolaridad, $34 \%$ algún grado de primaria, $22 \%$ algún grado de secundaria, $20 \%$ algún grado de preparatoria, $14 \%$ algún grado de universidad y $2 \%$ algún estudio de posgrado. Respecto a la ocupación el mayor porcentaje se dedica al hogar (38\%), mientras que otra parte se dedica al comercio o al estudio (29\%). De los encuestados, $90 \%$ manifiesta apreciar modificaciones en el clima de la región, como incremento en la temperatura, disminución en lluvias, aumento de heladas y retraso del régimen del temporal, que son los cambios más notorios.

"Yo siento que ahora está más fuerte el calor, en aquellos tiempos había muchos árboles, pinos en todas partes. Ahora se quemaron, los cortaron, se acabaron y por eso yo pienso que hace más calor. Cuando yo andaba en el cerro lloviznaba y me cubría en los árboles, ahora el cerro está pelón" (J. M. 50 años).

Del total de la población encuestada, $84 \%$ declaró conocer el término de $\mathrm{CC}$, al haberlo escuchado vía televisión o radio. Como responsable del CC, $60 \%$ de las respuestas obtenidas indican al ser humano, $25 \%$ lo considera como un fenómeno de carácter natural y $15 \%$ no sabe cuál sería la causa.

La información histórica de la región y la información cualitativa descrita por algunos entrevistados, muestran que el énfasis dado por los pobladores a la deforestación deriva de experiencias locales, particularmente su exposición a grupos criminales dedicados a la tala ilegal.

Al preguntarles si consideraban que los efectos del CC, podrían ocasionar repercusiones negativas en su salud, actividades económicas o bienestar social a mediano o largo plazo, el $100 \%$ de las respuestas fueron afirmativas. Además, agregaron que existe falta de información y claridad sobre las causas y 
CUADRO II. VARIABLES EMPLEADAS EN ELANÁLISIS DE COMPONENTES PRINCIPALES CATEGÓRICOS. SE DESCRIBE EL TIPO Y LAS CARACTERÍSTICAS DEL CÓMO SE INCORPORÓ EN EL ANÁLISIS

\begin{tabular}{|c|c|c|}
\hline Variable & Tipo & Observación \\
\hline Edad & Intervalos & La edad como se menciona \\
\hline Sexo & Nominal & $1=$ femenino $; 2$ = masculino \\
\hline Ocupación & Nominal & $\begin{array}{l}1=\text { jubilado o pensionado; } 2=\text { profesionista; } 3=\text { trabaja por su cuenta; } 4=\text { empleado } ; 5= \\
\text { campo; } 6=\text { artesano; } 7=\text { comerciante; } 8=\text { estudiante } 9=\text { hogar }\end{array}$ \\
\hline Escolaridad & Nominal & $\begin{array}{l}0=\sin \text { escolaridad; } 1=\text { primaria } ; 2=\text { secundaria; } 3=\text { preparatoria } ; 4=\text { universidad } ; 5= \\
\text { posgrado }\end{array}$ \\
\hline Localidad & Nominal & $1=$ Chilchota $;=$ Zopoco $3=$ Tanaquillo; $4=$ Carapan; $5=$ Ichan \\
\hline Hablante lengua indígena & Nominal & $1=\mathrm{no} ; 2=$ sí \\
\hline Principales problemas & Nominal & $\begin{array}{l}0=\text { ninguna respuesta; } 1=\text { pobreza } 2=\text { escasez de alimentos; } 3=\text { escasezde agua } 4=\text { falta } \\
\text { de acceso a la educación; } 5=\text { cambio climático; } 6=\text { crisis económica; } 7=\text { violencia; } 8= \\
\text { desempleo; } 9=\text { corrupción; } 10=\text { otro; } 11=1+2+3 ; 12=1+2+4 ; 13=1+2+5 ; 14=1+2+6 ; \\
15=1+2+7 ; 16=1+2+8 ; 17=1+2+9 ; 18=1+3+4 ; 19=1+3+5 ; 20=1+3+6 ; 21=1+3+7 ; \\
22=1+3+8 ; 23=1+3+9 ; 24=1+4+5 ; 25=1+4+6 ; 26=1+4+7 ; 27=1+4+8 ; 28=1+4+9 ; \\
29=1+5+6 ; 30=1+5+7 ; 31=1+5+8 ; 32=1+5+9 ; 33=1+6+7 ; 34=1+6+8 ; 35=1+6+9 ; \\
36=1+7+8 ; 37=1+7+9 ; 38=1+8+9 ; 39=2+3+5 ; 40=2+3+6 ; 41=2+4+7 ; 42=2+5+7 ; \\
43=2+6+8 ; 44=2+8+9 ; 45=3+4+6 ; 46=3+4+7 ; 47=3+5+6 ; 48=3+5+7 ; 49=3+5+9 ; \\
50=3+6+7 ; 51=3+6+8 ; 52=3+7+8 ; 53=3+7+9 ; 54=3+8+9 ; 55=3+9+10 ; 56=4+5+6 ; \\
57=4+5+8 ; 58=4+5+9 ; 59=4+6+8 ; 60=4+7+8 ; 61=5+6+7 ; 62=5+6+8 ; 63=5+6+9 ; \\
64=5+7+8 ; 65=5+7+9 ; 66=5+8+9 ; 67=6+7+8 ; 68=6+7+9 ; 69=6+8+9 ; 70=7+8+9 ; \\
71=1+9+10 ; 72=3+8+10 ; 73=2+6+10 ; 74=5+9+10 ; 75=8 ; 76=1+2+3+4+5+6+7+8+9 ;\end{array}$ \\
\hline
\end{tabular}

\begin{tabular}{lll}
\hline Percepción de temperatura & Nominal & $1=$ disminuido; $2=$ aumentado; $3=$ igual \\
\hline Percepción de lluvias & Nominal & $1=$ disminuido; $2=$ aumentado; $3=$ igual \\
\hline Percepción de heladas & Nominal & $1=$ disminuido; $2=$ aumentado; $3=$ igual \\
\hline Fenómenos climáticos locales & Nominal & $0=$ ninguno; $1=$ disminución de lluvias; $2=$ aumento de temperatura; $3=$ heladas más \\
& intensas; $4=$ vientos más intensos; $5=$ sequía o escasez de agua; $6=$ cambio en la duración \\
& de las estaciones; $7=1+2 ; 8=1+2+3 ; 9=1+2+3+4 ; 10=1+2+3+4+5+6 ; 11=1+2+3+4+5 ;$ \\
& $12=1+2+3+4+6 ; 13=1+2+5+6 ; 14=1+2+5 ; 15=1+2+3+6 ; 16=1+2+3+5+6 ; 17=1+4+6 ;$ \\
& $18=1+2+6 ; 19=1+2+4+5+6 ; 20=1+6 ; 21=1+3 ; 22=1+4+5 ; 23=1+2+4+6 ; 24=1+2+4 ;$ \\
& $25=1+5 ; 26=1+3+5 ; 27=1+5+6 ; 28=1+2+3+5 ; 28=1+2+4+5 ; 29=1+3+5+6 ; 30=2+4 ;$ \\
& $31=2+5 ; 32=2+3 ; 33=2+4+6 ; 34=3+4 ; 35=3+4+6 ; 36=2+6 ; 37=2+3+5+6 ; 38=2+3+5 ;$ \\
& $39=3+5 ; 40=2+3+6 ; 41=5+6 ; 42=4+6 ; 43=2+5+6 ; 44=4+5 ;$ \\
\hline
\end{tabular}

Cambios en temporalidad de Nominal $1=$ no; 2 =sí; 3 = no sé

lluvias

\begin{tabular}{lll}
\hline $\begin{array}{l}\text { Término cambio climático } \\
\text { (CC) }\end{array}$ & Nominal & $1=$ no; $2=$ sí \\
\hline Medio informativo & Nominal & $\begin{array}{l}1=\text { televisión; } 2=\text { radio; } 3=\text { escuela; } 4=\text { internet; } 5=\text { periódico; } 6=\text { amigos o } \\
\text { familiares; } 7=\text { otro }\end{array}$ \\
\hline Alcance del CC & Nominal & $1=$ local; $2=$ nacional; $3=$ mundial \\
\hline Causas del CC & Nominal & $1=$ ocurre de manera natural; $2=$ provocado por el ser humano; $3=$ no sé \\
\hline Responsables del CC & Nominal & $1=$ las industrias; $2=$ toda la sociedad; $3=$ el gobierno \\
\hline $\begin{array}{l}\text { Acciones que contribuyen el } \\
\text { CC }\end{array}$ & Nominal & $1=$ no; $2=$ sí \\
\hline Riesgos personales al CC & Nominal & $1=$ no; $2=$ sí; $3=$ no sé \\
\hline Información sobre el CC & Nominal & $1=$ no; $2=$ sí \\
\hline
\end{tabular}


los efectos del CC en su comunidad. A partir de los resultados del análisis multivariado, de acuerdo con los valores propios para cada iteración, se obtuvieron cantidades arriba de $1(\geq 5.48)$ lo que hace conveniente el análisis para determinar el porcentaje de varianza. El porcentaje de varianza acumulado en las primeras dimensiones fue como máximo $33.3 \%$, lo cual es un valor relativamente bajo (Cuadro III), pero consistente con estudios donde se involucran este tipo de variables nominales (Linting et al. 2007).

CUADRO III. RESUMEN DEL ANÁLISIS DE COMPONENTES PRINCIPALES CATEGÓRICOS APLICADO EN LAS ENCUESTAS

\begin{tabular}{cccc}
\hline Dimensiones & $\begin{array}{c}\text { Alfa de } \\
\text { Cronbach }\end{array}$ & $\begin{array}{c}\text { Varianza total } \\
\text { (Valor eigen) }\end{array}$ & $\begin{array}{c}\% \text { acumulado } \\
\text { varianza }\end{array}$ \\
\hline 1 & 0.781 & 3.9 & 20.3 \\
2 & 0.629 & 2.5 & 13.0 \\
Total & 0.889 & 6.3 & 33.3 \\
\hline
\end{tabular}

Dentro de la matriz de correlación (Cuadro IV), la relación más alta se obtuvo entre localidad y lengua indígena (0.905), lo que significa que existen diferencias culturales importantes aún en un espacio pequeño de varias comunidades aparentemente intercomunicadas. De igual manera, se presentan otras correlaciones significativas como ocupación y escolaridad que están inversamente relacionadas $(-0.648)$ y destaca para los fines de este estudio, las variables principales problemas y cambio climático (0.514), donde se encuentra una cierta asociación de causalidad.

El aporte de los componentes al modelo CAPT$\mathrm{CA}$, muestra que la primera dimensión separa, con valores positivos las variables: localidad (0.803), lengua indígena (0.704), ocupación (0.563) y cambios climáticos locales (0.537). Mientras que de manera negativa están las variables: principales problemas $(-0.799)$ y CC $(-0.656)$ (Cuadro V). La segunda dimensión se relaciona positivamente con la edad (0.757) y la ocupación (0.507) y negativamente con los fenómenos climáticos locales (0.587).

En la figura 2, se destacan comunidades representadas hacia el lado derecho donde se tiene una importante cantidad de gente con habla indígena, mientras que la comunidad de Chilchota, más mestiza, se encuentra hacia la izquierda. Asimismo, se puede observar que del lado izquierdo están aquellas comunidades más familiarizadas con el término y que de alguna manera lo relacionan con los principales problemas.

La figura 3 representa el comportamiento de la temperatura media anual de 1968-2007. En este periodo es evidente una tendencia hacia el aumento de la temperatura, con un incremento promedio de $0.5^{\circ} \mathrm{C}$.

Sobre la precipitación, se muestra una marcada tendencia a la disminución de la misma. De un promedio de $980 \mathrm{~mm}$ anuales a inicios de 1970, a casi $830 \mathrm{~mm}$ a finales de 2007, es decir en promedio llueve $150 \mathrm{~mm}$ anuales menos (Fig. 3).

En relación con lo percibido por los agricultores sobre los cambios climáticos y sus transformaciones en las prácticas de cultivo locales, encontramos que las precipitaciones se desfasaron de los calendarios agrícolas normales y que también son menores comparadas con años anteriores. Los encuestados manifiestan que los cambios en los regímenes de precipitación han modificado los calendarios agrícolas de siembra en la región. De acuerdo con los agricultores, en los últimos años las lluvias se retrasaron, por lo general se presentaban a mediados del mes de mayo y actualmente se manifiestan a finales de junio y en ocasiones a mediados de julio. Lo anterior ocasiona que los productores retrasen la fecha del inicio de siembra del maíz y también se ven afectados por las heladas que se adelantan en el tiempo.

"Hace 15 años estaba bien el tiempo, ahora llueve menos. Uno no puede tantear como viene el tiempo. Más antes llovía en mayo, por eso la gente sembraba mucho maíz. Pero ahora no quiere llover, sembramos tantita flor y no nació nada por falta de agua" (A.S. 80 años).

"Las heladas están cambiando, pues ahora incluso se están presentando en el mes de octubre, como pasó en Cheranástico (Comunidad indígena cercana a Chilchota), en este lugar la helada recién cayó en el mes de octubre y la siembra se perdió" (E.M. 53 años).

La variación en el clima, sobre todo el relacionado con el aumento de la temperatura, ha provocado que algunos productores modifiquen sus horarios de trabajo.

"Y un calorón que hace, como que el calor va en aumento. Antes el calor no se sentía así. Por allá en abril y mayo cómo irá a estar si en enero o febrero hace calor. Por eso para la cruza yo salgo a las 5 de la mañana y como a las 10 me vengo, porque el calor se siente refeo y luego la polvadera que se levanta" (L.L. 62 años).

\section{DISCUSIÓN}

Al referirnos a la influencia que los medios de comunicación tienen ante estos fenómenos, nuestros datos contrastan con un estudio realizado por Byg y 


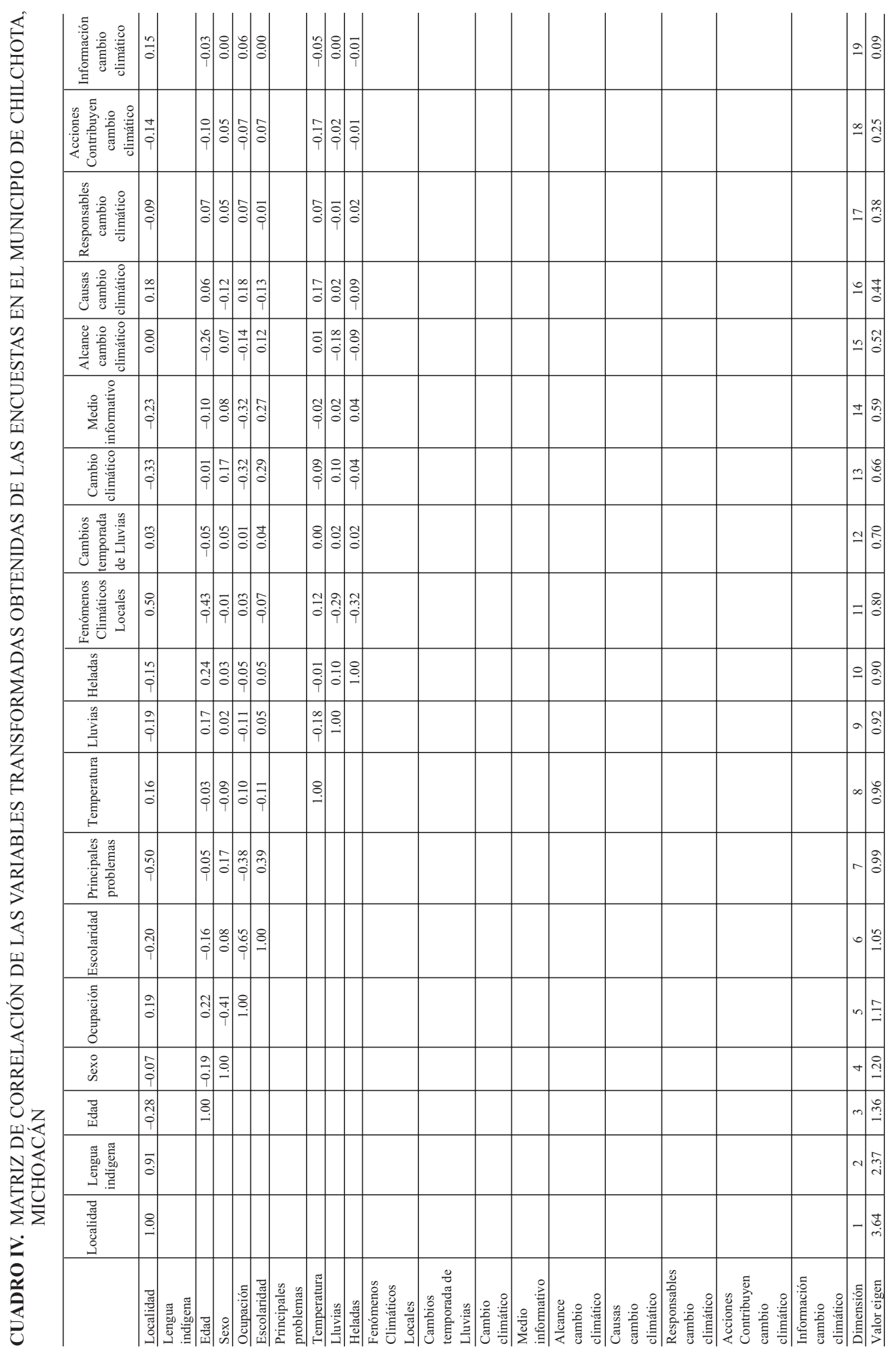


CUADRO V. APORTE A LAS DOS PRIMERAS DIMENSIONES DEL ANÁLISIS DE COMPONENTES PRINCIPALES CATEGÓRICOS

\begin{tabular}{lrr}
\hline & \multicolumn{2}{c}{ Dimensión } \\
\cline { 2 - 3 } & \multicolumn{1}{c}{1} & \multicolumn{1}{c}{2} \\
\hline Localidad & 0.803 & -0.367 \\
Lengua indígena & 0.743 & -0.407 \\
Edad & -0.126 & 0.757 \\
Sexo & -0.279 & -0.354 \\
Ocupación & 0.563 & 0.507 \\
Escolaridad & -0.478 & -0.426 \\
Principales problemas & -0.779 & -0.158 \\
Temperatura & 0.287 & 0.007 \\
Lluvias & -0.282 & 0.314 \\
Heladas & -0.202 & 0.332 \\
Fenómenos Climáticos locales & 0.537 & -0.587 \\
Cambios temporada de lluvias & 0.041 & -0.042 \\
Cambio climático & -0.656 & -0.192 \\
Medio informativo & -0.440 & -0.195 \\
Alcance cambio climático & -0.123 & -0.497 \\
Causas cambio climático & 0.422 & 0.289 \\
Responsables cambio climático & 0.121 & 0.178 \\
Acciones Contribuyen cambio climático & -0.231 & -0.150 \\
Información cambio climático & 0.171 & 0.006 \\
\hline
\end{tabular}

Salick (2009) en la provincia tibetana. En el estudio se señala que los aldeanos de esta región no habían escuchado hablar del fenómeno de cambio climático global y que consideraban que estos cambios

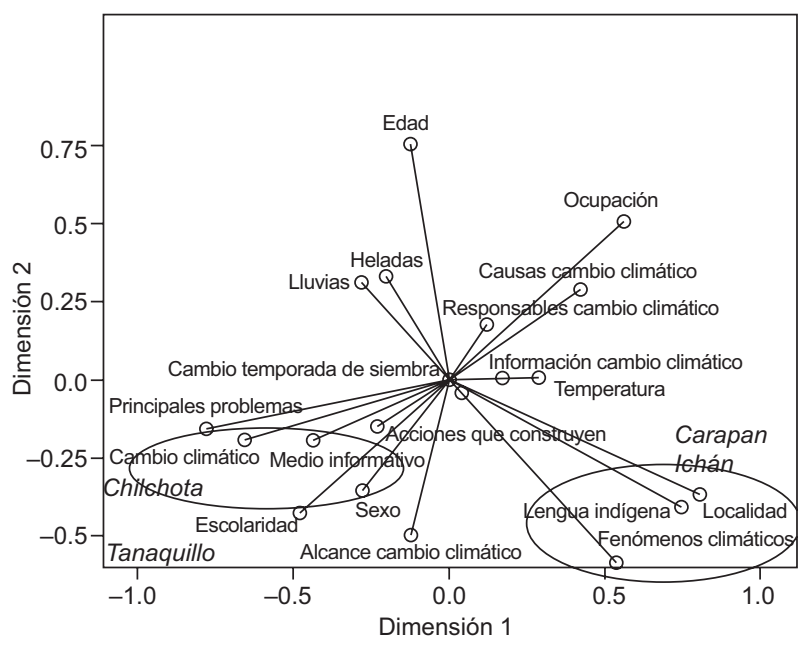

Fig. 2. Análisis de componentes principales categóricos

eran fenómenos locales, situación que los autores atribuyen a la poca importancia y conciencia que para ese entonces se le estaba dando a las cuestiones referentes al tema, incluso por los medios de comunicación. Por su parte, Rudiak-Gould (2014) señala la "apertura" del conocimiento local sobre el CC frente a la difusión de programas de divulgación de la ciencia, de modo que las explicaciones "locales" incorporen en realidad conocimientos científicos, lo que hace difícil distinguir entre ambos. Otro estudio que valora el
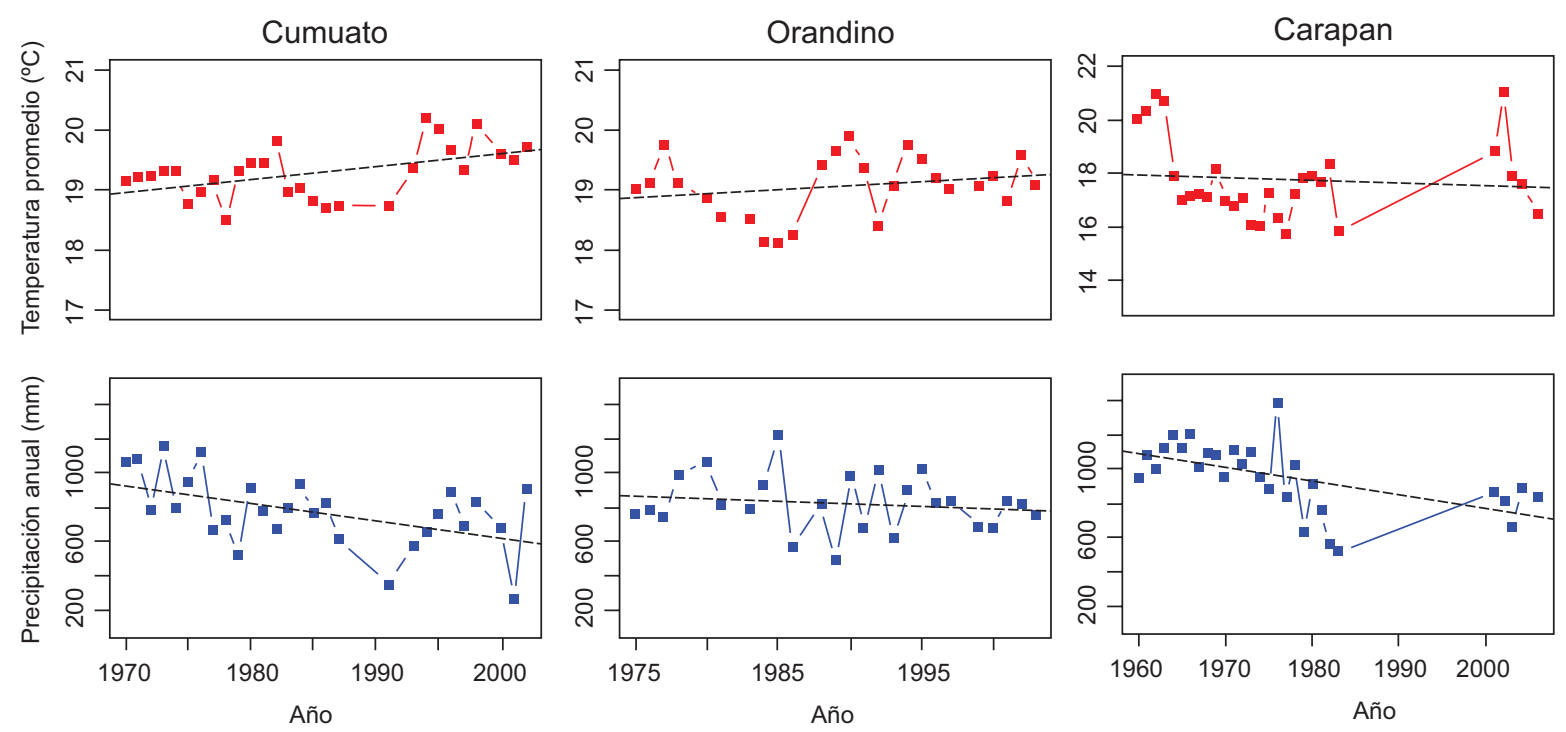

Fig. 3. Comportamiento de la temperatura y precipitación media anual para el periodo 1968-2007, dentro de la zona de estudio 
efecto de transmitir información científica sobre el CC a poblaciones indígenas es el de Fernández et al. (2015), en el que los autores encontraron que no cambiaba mucho la percepción de la población al respecto, pues el "conocimiento experiencial" supera al conocimiento descriptivo. Los efectos mencionados, ya sea sentidos o percibidos en esta región, son congruentes con lo advertido por el IPCC (1995), donde se pronosticó un incremento en la temperatura. A medida que la temperatura continúe elevándose, el impacto en la agricultura será significativo.

Ishaya (2008), realizó un estudio en Kaduna State, Nigeria, en el que los resultados revelaron que las poblaciones indígenas percibieron que el ambiente y el clima han ido cambiando con los años debido a diversas actividades humanas. Indicaron que la amenaza del CC recae sobre la salud, el suministro de alimentos y la pérdida de biodiversidad. Esta misma percepción se observa en la comunidad purépecha, en la que señalan que los $\mathrm{CC}$ son un factor de riesgo a la salud, al ambiente local y a su seguridad alimentaria.

Echeverri (2009), menciona la poca importancia que se le ha dado a los aspectos sociales en el tema de CC, a pesar de que los pobres del mundo y los pueblos indígenas son los más vulnerables a dicho fenómeno y que el IPCC (2007), apenas los menciona en su informe. En su estudio, el autor encuentra una percepción generalizada en las comunidades de la Amazonía colombiana, que desde 1990 notan evidentes alteraciones en el clima (particularmente en la estacionalidad), dichos cambios se han mantenido constantes desde 2005. Este resultado es consistente con lo percibido por la comunidad indígena purépecha de este estudio, al hacer énfasis en la apreciación de cambios severos en la temperatura y en las precipitaciones de manera estacional. Byg y Salick (2009) aseguran que sólo las comunidades a nivel local pueden evaluar, interpretar o sentir los efectos del CC y que estas observaciones no se pueden sustituir por modelos o mediciones científicas. En este estudio, las percepciones reportadas coinciden en general con dichos autores, al encontrar ese factor de evaluación e interpretación sobre los efectos del $\mathrm{CC}$ en los indígenas purépechas.

En la zona indígena Zoque en México, Sánchez y Lazos (2011) definieron cómo los campesinos de esa región perciben una disminución en las precipitaciones y un aumento en la temperatura. Lo anterior, debido a que estos factores están relacionados con modificaciones en la temporada de siembra de maíz y la introducción de cultivos que, por lo general, sólo se localizan en las regiones cálidas. Esto es similar a lo reportado en este estudio para la zona indígena purépecha, sin embargo no se han introducido aún nuevos cultivos por dichas alteraciones climáticas.

Cruz (2011) se enfocó en la cosmovisión de los habitantes de pueblos mazahuas del norte del Estado de México, en relación al cultivo de maíz y de los cambios que ellos han notado en el patrón anual de lluvias. Su investigación permitió comprobar que la mayoría de los entrevistados se han visto obligados a realizar los trabajos de la milpa haciendo ajustes en el tradicional calendario agrícola, lo que ha dado lugar a una tricotomía entre el tiempo meteorológico, el tiempo ritual y el tiempo de labor agrícola. A lo cual los pobladores se están tratando de acostumbrar. Además, a todo ello se le suma la notable disminución, año con año, de la producción agrícola con sus respectivas consecuencias económicas. En la comunidad indígena purépecha, estas condiciones aquí citadas son reflejadas en los testimonios encontrados por los entrevistados.

Un ejemplo más de lo anterior lo reporta Solís y Salvatierra (2013) en su trabajo sobre la percepción del CC en áreas destinadas voluntariamente a la conservación de recursos naturales en comunidades indígenas de Oaxaca y Chiapas. Menciona cómo los pobladores de estos grupos indígenas han escuchado acerca del CC por medio de noticias, diálogos con gente externa a su comunidad o en las juntas que realizan internamente. Sin embargo, se refieren a ellos mismos como causantes de esos cambios que afectan su subsistencia y bienestar. No utilizan el término "cambio climático", ellos viven estas consecuencias desde hace años, sin poder darles una explicación a partir de sus conocimientos tradicionales. Del mismo modo, lo encontrado en este estudio es similar a lo reportado por Soares y García (2014), sobre los campesinos indígenas de la cuenca de Jovel, Chiapas que perciben cambios profundos en algunos eventos meteorológicos. De manera particular en la periodicidad de las heladas y del periodo de lluvias, así como del incremento en la intensidad de las granizadas, con impactos heterogéneos sobre sus condiciones de vida. Ellos mismos son uno de los actores que reciben más impactados por esta variabilidad climática, debido a su dependencia de los recursos naturales, de sus vulnerables medios de vida y de su pobreza.

Es palpable cómo los efectos locales del cambio climático se perciben cotidianamente en el modo de vivir de la comunidad indígena purépecha. Se puede observar que las actividades económicas ligadas al campo están sujetas a procesos de adaptación climática. 
En muchos países, en especial la gente con bajos niveles económicos, son ahora forzados a vivir en áreas expuestas y marginales (áreas inundables, zonas de laderas expuestas y tierras áridas o semiáridas), lo que los pone en riesgo de sufrir los impactos negativos del CC. Para esta gente, aún los cambios menores en el clima pueden tener un impacto devastador en sus vidas y en sus fuentes de sustento. Sobre todo para aquellos agricultores de subsistencia ubicados en ambientes frágiles, donde se espera que estos cambios podrían propiciar una reducida productividad. La vulnerabilidad de estos agricultores aumenta, pues dependen de cultivos potencialmente afectados (maíz, frijol, papas, arroz, etc.) para su seguridad alimentaria.

\section{CONCLUSIONES}

En relación con los estudios en comunidades indígenas en otras partes del mundo y en México, esta investigación confirma la relación entre los saberes campesinos derivados de adaptaciones sociales a condiciones ambientales regionales. Así como, la posibilidad de una percepción de alteraciones climáticas según la interacción del entrevistado con el ambiente local, puesto que los habitantes de las comunidades indígenas fueron quienes asociaron las variaciones en el clima con acciones concretas que lleva a cabo el ser humano en su región.

La población indígena dentro del municipio de Chilchota percibió cambios locales en el clima atribuidos a diversas acciones que el ser humano ha desarrollado en los últimos años (tala de árboles, quema de basura, contaminación, etc.). Sin embargo, no asociaron dichos cambios de manera inmediata con el fenómeno de CC. No obstante, sí le atribuyen importancia por el riesgo a perder su forma de vida que depende directamente de los recursos naturales locales.

Lo más valioso es la manera en que los entrevistados han interpretado sus percepciones sobre alteraciones climáticas locales con conflictos de la historia regional reciente y aún vigente en la actualidad: la corrupción y la tala ilegal de amplias zonas que habita la población purépecha.

Las localidades predominantemente indígenas (Carapan, Ichán y Zopoco) identificaron en mayor medida las causas y consecuencias del $\mathrm{CC}$, lo que asociaron a sus propias actividades de carácter agrícola. Por otra parte, la comunidad de Chilchota con una población mayormente mestiza, admite que las alteraciones climáticas pudieran afectar su bienestar a un corto, mediano o largo plazo.
De acuerdo con los agricultores, en los últimos años las lluvias se han retrasado, lo que ha ocasionado que retarden la fecha del inicio de siembra del cultivo de maíz y se vean afectados por las heladas. Por lo cual y como medida de adaptación, los calendarios agrícolas de siembra de la región se han ajustado.

Los estudios sobre la percepción del CC en comunidades indígenas y medios de comunicación, en la mayoría de los casos están orientados a conocer el avance de ese concepto sobre la población. Sin embargo, consideramos que dichos estudios parten de un modelo único de desarrollo y no se cuestionan que las poblaciones indígenas en realidad mantienen principios de desarrollo distintos. Sería más provechoso indagar sobre el significado de un proceso de cambio ambiental global, como lo es el CC, desde la perspectiva de estas comunidades indígenas, identificando las relaciones económicas y políticas del ámbito nacional e internacional que las afectan, para vincularlas con las alteraciones climáticas locales.

\section{AGRADECIMIENTOS}

Este proyecto se realizó gracias al financiamiento otorgado por el Instituto Politécnico Nacional. Se reconoce el apoyo proporcionado por la comisión de cuenca del río Duero.

\section{REFERENCIAS}

AEMA (2015). El medio ambiente en Europa: Estado y perspectivas 2015-Informe de síntesis. Agencia Europea de Medio Ambiente, Copenhague, Dinamarca, 178 pp. DOI: $10.2800 / 50970$

Altieri M. y Clara N. (2008). Los impactos de cambio climático sobre las comunidades campesinas y de agricultores tradicionales y sus respuestas adaptativas. Rev. Inv. Agroecol. 3 (7), 7-23.

Arnell N. W. (1999). The effect of climate change on hydrological regimes in Europe: A continental perspective. Glob. Environ.Chang. 9 (1), 5-23.

Barnett T., Adam J. y Lettenmaier D. (2005). Potential impacts of a warming climate on water availability in snow-dominated regions. Nature 438 (7066), 303-309. DOI: 10.1038 /nature04141

Beniston M. (2003). Climatic change in mountain regions: a review of possible impacts. Clim. Chang. 59 (1-2), 5-31. DOI: 10.1023/A:1024458411589

Brown O. (2008). Migración y cambio climático. Serie 31. Organización internacional para las migraciones, Ginebra, Suiza, 54 pp. 
Byg A. y Salick J. (2009). Local perspectives on a global phenomenon climate change in eastern Tibetan Villages. Global Environ. Chang. 19 (2), 156-166.

DOI: 10.1016/j.gloenvcha.2009.01.010

Cronbach L. J. (1951). Coefficient alpha and the internal structure of test. Psychometrika 16 (3), 297-334. DOI: $10.1007 / \mathrm{BF} 02310555$

Cruz L. M. (2011). Comparación del ciclo agrícola actual con el de hace unos diez años en San Juan Jalpa municipio de San Felipe del Progreso, Estado de México: evidencia de adaptación al cambio climático. Ra Ximhai 7 (1), 95-106.

Deressa T., Hassan R. y Ringler C. (2011). Perception of and adaptation to climate change by farmers in the Nile basin of Ethiopia. J. Agric. Sci. 149 (1), 23-31. DOI: $10.1017 / \mathrm{S} 0021859610000687$

Echeverri J.A. (2009). Pueblos indígenas y cambio climático: el caso de la Amazonía colombiana. Bull. Inst. Fr. Etudes Andin. 38 (1), 13-28. DOI:10.4000/bifea.2774

Fernández Ll. A., Méndez L.M., Díaz R.I., McBride M., Pyhälä A., Rosell M. A. y Reyes G. V. (2015). Links between media communication and local perceptions of climate change in an indigenous society. Clim. Chang. 131 (2), 307-320. DOI: 10.1007/s10584-015-1381-7

García E. (1973). Modificaciones al sistema de clasificación climática de Koppen, 2da. Edición. Instituto de Geografía. Universidad Nacional Autónoma de México, Ciudad de México, México, 98 pp.

Gay C. (2000). México: una visión hacia el siglo XXI. El cambio climático en México. Instituto Nacional de Ecología, Universidad Nacional Autónoma de México, US Country Studies Program, Ciudad de México, México, 220 pp.

Gay C., Estrada F., Conde C., Eakin H. y Villers L. (2006). Potential impacts of climate change on agriculture: a case of study of coffee production in Veracruz, Mexico. Clim. Chang. 79 (3-4), 259-288.

DOI: $10.1007 / \mathrm{s} 10584-006-9066-\mathrm{x}$

Giddens A. y Muñoz F. (2010). La política del cambio climático. Alianza, Madrid, España, 304 pp.

INEGI (2015). Encuesta intercensal, principales resultados. Instituto Nacional de Estadística Geografía e Informática. Ciudad de México, México, 92 pp.

IPCC (1990). Climate change. First report. The Scientific Assessment Intergovernmental Panel on Climate Change. Reino Unido y Nueva York, EUA, Cambridge, University Press, $571 \mathrm{pp}$.

IPCC (1995). Climate change. First report. The Scientific Assessment Intergovernmental Panel on Climate Change. Reino Unido y Nueva York, EUA. Cambridge, University Press, 73 pp.

IPCC (2014). Resumen para responsables de políticas. En: Cambio climático 2014: mitigación del cambio climático. Contribución del grupo de trabajo II al quinto informe de evaluación del grupo intergubernamental de expertos sobre el cambio climático. The Scientific Assessment Intergovernmental Panel on Climate Change, Ginebra, Suiza, 34 pp.

Ishaya S. y Abaje I. (2008). Indigenous people's perception on climate change and adaptation strategies in Jema' a local government area of Kaduna State, Nigeria. J. Geogr. Reg. Plann. 1 (8), 138-143.

Linting M., Meulman J., Groenen P. y Van der Kooij J. (2007). Nonlinear principal components analysis: introduction and application. Psychol. Methods 12 (3), 336-358. DOI: 10.1037/1082-989X.12.3.336

Linting M. y Van der Kooij A. (2012). Nonlinear principal components analysis with CATPCA: A tutorial. J. Pers. Assess. 94 (1), 12-25.

DOI: $10.1080 / 00223891.2011 .627965$

McDowell Z. (2010). Cambio climático: vulnerabilidad social y escasez de agua en comunidades indígenas campesinas de Bolivia. Agua Ambiente 3, 11-33.

Manisera M., Van Der Kooij A. y Dusseldorp E. (2010). Identifying the component structure of satisfaction scales by nonlinear principal components analysis. Qual. Technol. Quant. M. 7 (2), 97-115.

Maddison D. (2006). The perception of and adaptation to climate change in Africa. Pretoria, Sudáfrica: Discussion paper No. 10. Centre for Environmental Economics and Policy in Africa. University of Pretoria, Pretoria, Sudáfrica, $114 \mathrm{pp}$.

Marzano M. (2006). Changes in the weather: A Sri Lanka village case study. Appl. Anthropol. 13 (3), 63-76. DOI: 10.3167/aia.2006.130307

Nielsen J. y Reenberg A. (2010). Cultural barriers to climate change adaptation: A case study from northern Burkina Faso. Global Environ. Chang. 20 (1), 142-152. DOI: 10.1016/j.gloenvcha.2009.10.002

Osbahr H., Chasca T., Neil A., David S. y Thomas G. (2008). Effective livelihood adaptation to climate change disturbance: Scale dimensions of practice in Mozambique. Geoforum 39 (6), 1951-1964.

DOI: $10.1016 /$ j.geoforum.2008.07.010

Rudiak-Gould P. (2014). The influence of science communication on indigenous climate change perception: Theoretical and practical implications. Hum. Ecol. 42 (1), 75-86. DOI: 10.1007/s10745-013-9605-9

Salick J. y Byg A. (2007). Indigenous peoples and climate change. Oxford. Tyndall Centre for Climate Change Research. Oxford University, Oxford, Reino Unido, 32 pp.

Sánchez M. y Lazos Ch. (2010). Indigenous perception of changes in climate variability and its relationship with agriculture in a Zoque community of Chiapas, Mexico. Clim. Chang. 107 (3-4), 363-389.

DOI: $10.1007 / \mathrm{s} 10584-010-9972-9$ 
SEDESOL-CNEPDS (2015). Informe anual sobre la situación de pobreza y rezago social. Secretaría de Desarrollo Social - Consejo Nacional de Evaluación de la Política de Desarrollo Social, Ciudad de México, México, $15 \mathrm{pp}$.

SEMARNAT-CICC (2007). Estrategia nacional de cambio climático. Síntesis. Secretaría de Medio Ambiente y Recursos Naturales - Comisión Intersecretarial de Cambio Climático Ciudad de México, México, 16 pp.

Soares D. y García A. (2014). Percepciones campesinas indígenas acerca del cambio climático en la cuenca de Jovel, Chiapas, México. Cuad. Antrop. Soc. 39, 63-89.

Solís J. y Salvatierra B. (2013). Percepción social del cambio climático en áreas destinadas voluntariamente a la conservación en comunidades indígenas de Oaxaca y Chiapas. Revista Científica de Investigaciones Regionales 35 (1), 29-53.
Vedwan N. y Rhoades R. (2001). Climate change in the western Himalayas of India: a study of local perception and response. Clim. Res. 19 (2), 109-117. DOI: $10.3354 /$ cr019109

Walther G. R., Post E., Convey P., Menzel A., Parmesan C., Beebee T.J.C., Fromentin J. M., Guldberg O. H. y Bairlein F. (2002). Ecological responses to recent climate change. Nature 416, 389-395. DOI: 10.1038/416389a Zeijl E., Poel Y., Bois M., Ravesloot J. y Meulman J. (2000). The role of parents and peers in the leisure activities of young adolescents. J. Leisure Res. 32 (3), 281-301. 